\title{
Oscillation of fourth-order neutral differential equations with $p$-Laplacian like operators
}

\author{
Tongxing Li ${ }^{1 *}$, Blanka Baculíková ${ }^{2}$, Jozef Džurina ${ }^{2}$ and Chenghui Zhang ${ }^{3}$
}

This paper is dedicated to Professor Ivan Kiguradze

\author{
"Correspondence: \\ litongx2007@163.com \\ ${ }^{1}$ Qingdao Technological University, \\ Feixian, Shandong 273400, P.R. \\ China \\ Full list of author information is \\ available at the end of the article
}

\begin{abstract}
We study oscillatory behavior of a class of fourth-order neutral differential equations with a $p$-Laplacian like operator using the Riccati transformation and integral averaging technique. A Kamenev-type oscillation criterion is presented assuming that the noncanonical case is satisfied. This new theorem complements and improves a number of results reported in the literature. An illustrative example is provided.

MSC: $34 \mathrm{C} 10 ; 34 \mathrm{~K} 11$
\end{abstract}

Keywords: oscillation; fourth-order neutral differential equation; $p$-Laplace differential equation; noncanonical operator

\section{Introduction}

In this paper, we are concerned with oscillation of a class of fourth-order neutral differential equations with a $p$-Laplacian like operator

$$
\left(r(t)\left|z^{\prime \prime \prime}(t)\right|^{p-2} z^{\prime \prime \prime}(t)\right)^{\prime}+\sum_{i=1}^{l} q_{i}(t)\left|x\left(\tau_{i}(t)\right)\right|^{p-2} x\left(\tau_{i}(t)\right)=0,
$$

where

$$
z(t):=x(t)+a(t) x(\sigma(t))
$$

It is interesting to study equation (1.1) since the $p$-Laplace differential equations have applications in continuum mechanics as seen from [1]. Throughout, we assume that $p>1$ is a constant, $\mathbb{I}:=\left[t_{0}, \infty\right), r \in \mathrm{C}^{1}(\mathbb{I},(0, \infty)), r^{\prime}(t) \geq 0, a, \sigma, q_{i}, \tau_{i} \in \mathrm{C}(\mathbb{I}, \mathbb{R}), 0 \leq a(t)<1, q_{i}(t) \geq 0$, $i=1,2, \ldots, l, \sigma(t) \leq t, \lim _{t \rightarrow \infty} \sigma(t)=\infty$, there exists a function $\tau \in C^{1}(\mathbb{I}, \mathbb{R})$ such that $\tau(t) \leq \tau_{i}(t)$ for $i=1,2, \ldots, l, \tau(t) \leq t, \tau^{\prime}(t)>0$, and $\lim _{t \rightarrow \infty} \tau(t)=\infty$.

We use the notation $t_{-1}:=\min _{t \in\left[t_{0}, \infty\right)}\left\{\sigma(t), \tau_{1}(t), \tau_{2}(t), \ldots, \tau_{l}(t)\right\}$. By a solution of (1.1), we mean a function $x \in C\left(\left[t_{-1}, \infty\right), \mathbb{R}\right)$ which has the property $r\left|z^{\prime \prime \prime}\right|^{p-2} z^{\prime \prime \prime} \in \mathrm{C}^{1}(\mathbb{I}, \mathbb{R})$ and satisfies (1.1) on $\mathbb{I}$. We consider only those solutions $x$ of (1.1) which satisfy $\sup \left\{|x(t)|: t \geq t_{*}\right\}>0$ for all $t_{*} \geq t_{0}$ and tacitly assume that (1.1) possesses such solutions. A solution $x$ of (1.1) is called oscillatory if it has arbitrarily large zeros on $\mathbb{I}$; otherwise, it is said to be nonoscillatory. Equation (1.1) is termed oscillatory if all its solutions oscillate.

Fourth-order differential equations naturally appear in models concerning physical, biological, and chemical phenomena; see [2]. In mechanical and engineering problems,

๑2014 Li et al.; licensee Springer. This is an Open Access article distributed under the terms of the Creative Commons Attribution License (http://creativecommons.org/licenses/by/2.0), which permits unrestricted use, distribution, and reproduction in any medium, provided the original work is properly cited. 
questions related to the existence of oscillatory solutions play an important role. During the past few years, there has been constant interest in obtaining sufficient conditions for oscillatory and nonoscillatory properties of different classes of fourth-order differential equations. We refer the reader to [3-21] and the references cited therein. Parhi and Tripathy $[12,13]$ and Thandapani and Savitri [15] studied a fourth-order neutral differential equation

$$
\left(r(t)(x(t)+p(t) x(\sigma(t)))^{\prime \prime}\right)^{\prime \prime}+q(t) x(\tau(t))=0 .
$$

Most oscillation results reported in $[6,7,9,18]$ for $(1.1)$ and its particular cases have been obtained under the assumption that

$$
R\left(t_{0}\right)=\infty,
$$

where

$$
R(t):=\int_{t}^{\infty} \frac{\mathrm{d} s}{r^{1 /(p-1)}(s)} .
$$

The analogue for $(1.1)$ in case $a(t)=0$ has been studied in $[10,16,17,19-21]$ under the condition that

$$
R\left(t_{0}\right)<\infty,
$$

which is called a noncanonical case. Assuming (1.3), a question regarding the oscillation and asymptotic behavior of solutions to (1.1) in the case

$$
p=2, \quad l=1, \quad 0 \leq a(t) \leq a_{1}<1, \quad \text { and } \quad \tau_{1}(t) \leq t
$$

has been studied by Li et al. [11]. Note that [11, Theorem 2.2] ensures that every solution $x$ of the studied equation is either oscillatory or tends to zero as $t \rightarrow \infty$ and, unfortunately, cannot distinguish solutions with different behaviors.

It should be noted that research in this paper is strongly motivated by the recent paper [11]. The purpose of this paper is to establish a Kamenev-type theorem which guarantees that all solutions of equation (1.1) are oscillatory in the case where (1.3) holds and without requiring conditions (1.4). In the sequel, all functional inequalities are assumed to hold for all $t$ large enough.

\section{Main results}

We begin with the following lemma.

Lemma 2.1 (See [14]) Let $f \in \mathrm{C}^{n}\left(\mathbb{I}, \mathbb{R}^{+}\right)$. Assume that $f^{(n)}$ is eventually of one sign for all large $t$, and there exists a $t_{1} \geq t_{0}$ such that $f^{(n)}(t) f^{(n-1)}(t) \leq 0$ for all $t \geq t_{1}$. Then, for every constant $\lambda \in(0,1)$, there exist a $t_{\lambda} \in\left[t_{1}, \infty\right)$ and a constant $M>0$ such that

$$
f(\lambda t) \geq M t^{n-1}\left|f^{(n-1)}(t)\right|
$$

for all $t \in\left[t_{\lambda}, \infty\right)$. 
Lemma 2.2 (See [4, Lemma 2.2.3]) Let $f$ be as in Lemma 2.1. If $\lim _{t \rightarrow \infty} f(t) \neq 0$, then, for every constant $k \in(0,1)$, there exists a $t_{k} \in\left[t_{1}, \infty\right)$ such that

$$
f(t) \geq \frac{k}{(n-1) !} t^{n-1}\left|f^{(n-1)}(t)\right|
$$

for all $t \in\left[t_{k}, \infty\right)$.

Theorem 2.3 Assume (1.3) and let one of the following conditions hold:

$$
\int_{t_{0}}^{\infty} R(s) \mathrm{d} s=\infty
$$

and

$$
\int_{t_{0}}^{\infty} \int_{u}^{\infty} R(s) \mathrm{d} s \mathrm{~d} u=\infty
$$

Suppose also that there exist functions $\rho \in C^{1}(\mathbb{I},(0, \infty)), H, \varrho \in C(\mathbb{D}, \mathbb{R})$, where $\mathbb{D}=\{(t, s)$ : $t \geq s \geq t_{0}$ \} such that

$$
H(t, t)=0, \quad t \geq t_{0}, \quad H(t, s)>0, \quad t>s \geq t_{0},
$$

and $H$ has a nonpositive continuous partial derivative $\partial H / \partial s$ satisfying, for all sufficiently large $T \geq t_{0}$, for some constant $\lambda \in(0,1)$, and for all constants $M>0$,

$$
\begin{gathered}
\limsup _{t \rightarrow \infty} \frac{1}{H(t, T)} \int_{T}^{t}\left[H(t, s) \rho(s) \sum_{i=1}^{l} q_{i}(s)\left(1-a\left(\tau_{i}(s)\right)\right)^{p-1}\right. \\
\left.-\frac{1}{p^{p}} \frac{r(s)\left(\varrho_{+}(t, s)\right)^{p}}{\left(\lambda M \tau^{\prime}(s) \tau^{2}(s) \rho(s)\right)^{p-1}}\right] \mathrm{d} s=\infty
\end{gathered}
$$

where

$$
\varrho_{+}(t, s):=\max \{0, \varrho(t, s)\}
$$

and

$$
\frac{\partial H(t, s)}{\partial s}+\frac{\rho^{\prime}(s)}{\rho(s)} H(t, s)=\frac{\varrho(t, s)}{\rho(s)}(H(t, s))^{(p-1) / p} .
$$

If there exist functions $\delta \in \mathrm{C}^{1}(\mathbb{I},(0, \infty)), K, \xi \in \mathrm{C}(\mathbb{D}, \mathbb{R})$ such that

$$
K(t, t)=0, \quad t \geq t_{0}, \quad K(t, s)>0, \quad t>s \geq t_{0},
$$

and $K$ has a nonpositive continuous partial derivative $\partial K / \partial$ satisfying, for all sufficiently large $T \geq t_{0}$ and for some constant $k \in(0,1)$,

$$
\begin{aligned}
\limsup _{t \rightarrow \infty} \int_{T}^{t}\left[K(t, s) \delta(s)\left(\frac{k \tau^{2}(s)}{2}\right)^{p-1} \sum_{i=1}^{l} q_{i}(s)\left(1-a\left(\tau_{i}(s)\right)\right)^{p-1}\right. \\
\left.-\frac{r(s)\left(\xi_{+}(t, s)\right)^{p}}{p^{p} \delta^{p-1}(s)}\right] \mathrm{d} s>0,
\end{aligned}
$$


where

$$
\xi_{+}(t, s):=\max \{0, \xi(t, s)\}
$$

and

$$
\frac{\partial K(t, s)}{\partial s}+\frac{\delta^{\prime}(s)}{\delta(s)} K(t, s)=-\frac{\xi(t, s)}{\delta(s)}(K(t, s))^{(p-1) / p},
$$

then equation (1.1) is oscillatory.

Proof Let $x$ be a nonoscillatory solution of (1.1). Without loss of generality, we may assume that $x$ is eventually positive. Equation (1.1) implies that there exists a $t_{1} \geq t_{0}$ such that the following three possible cases hold for all $t \geq t_{1}$ :

(1) $z(t)>0, z^{\prime}(t)<0, z^{\prime \prime}(t)>0, z^{\prime \prime \prime}(t)<0,\left(r\left|z^{\prime \prime \prime}\right|^{p-2} z^{\prime \prime \prime}\right)^{\prime}(t) \leq 0$

(2) $z(t)>0, z^{\prime}(t)>0, z^{\prime \prime \prime}(t)>0, z^{(4)}(t) \leq 0,\left(r\left|z^{\prime \prime \prime}\right|^{p-2} z^{\prime \prime \prime}\right)^{\prime}(t) \leq 0$;

(3) $z(t)>0, z^{\prime}(t)>0, z^{\prime \prime}(t)>0, z^{\prime \prime \prime}(t)<0,\left(r\left|z^{\prime \prime \prime}\right|^{p-2} z^{\prime \prime \prime}\right)^{\prime}(t) \leq 0$.

We consider each of these cases separately.

Case 1. Assume that (1) is satisfied. Noting that $r\left(-z^{\prime \prime \prime}\right)^{p-1}$ is nondecreasing, we have, for $s \geq t \geq t_{1}$

$$
r^{1 /(p-1)}(s) z^{\prime \prime \prime}(s) \leq r^{1 /(p-1)}(t) z^{\prime \prime \prime}(t) .
$$

Dividing the latter inequality by $r^{1 /(p-1)}(s)$ and integrating the resulting inequality from $t$ to $\iota, \iota \geq t \geq t_{1}$, we obtain

$$
z^{\prime \prime}(\iota) \leq z^{\prime \prime}(t)+r^{1 /(p-1)}(t) z^{\prime \prime \prime}(t) \int_{t}^{\iota} \frac{\mathrm{d} s}{r^{1 /(p-1)}(s)} .
$$

Passing to the limit as $\iota \rightarrow \infty$, we conclude that

$$
z^{\prime \prime}(t) \geq-r^{1 /(p-1)}(t) z^{\prime \prime \prime}(t) R(t)
$$

Hence, there exists a constant $c>0$ such that

$$
z^{\prime \prime}(t) \geq c R(t)
$$

Integrating (2.5) from $t_{1}$ to $t$, we have

$$
z^{\prime}(t)-z^{\prime}\left(t_{1}\right) \geq c \int_{t_{1}}^{t} R(s) \mathrm{d} s .
$$

This yields

$$
-z^{\prime}\left(t_{1}\right) \geq c \int_{t_{1}}^{t} R(s) \mathrm{d} s
$$

which contradicts (2.1). Next, integrating (2.5) from $t$ to $\infty$, we get

$$
-z^{\prime}(t) \geq c \int_{t}^{\infty} R(s) \mathrm{d} s .
$$


Integrating again from $t_{1}$ to $t$, we have

$$
-z(t)+z\left(t_{1}\right) \geq c \int_{t_{1}}^{t} \int_{u}^{\infty} R(s) \mathrm{d} s \mathrm{~d} u .
$$

This implies that

$$
z\left(t_{1}\right) \geq c \int_{t_{1}}^{t} \int_{u}^{\infty} R(s) \mathrm{d} s \mathrm{~d} u,
$$

which contradicts (2.2).

Case 2. Assume that (2) is satisfied and let $\lambda \in(0,1)$ be an arbitrary constant. Then, there exists a $t_{\lambda} \geq t_{1}$ such that, for all $t \geq t_{\lambda}, z(\lambda \tau(t))>0$. For $t \geq t_{\lambda}$, define

$$
\omega(t):=\rho(t) \frac{r(t)\left(z^{\prime \prime \prime}(t)\right)^{p-1}}{z^{p-1}(\lambda \tau(t))} .
$$

Then $\omega(t)>0$ for all $t \geq t_{\lambda}$, and

$$
\begin{aligned}
\omega^{\prime}(t)= & \rho^{\prime}(t) \frac{r(t)\left(z^{\prime \prime \prime}(t)\right)^{p-1}}{z^{p-1}(\lambda \tau(t))}+\rho(t) \frac{\left(r(t)\left(z^{\prime \prime \prime}(t)\right)^{p-1}\right)^{\prime}}{z^{p-1}(\lambda \tau(t))} \\
& -(p-1) \lambda \rho(t) \frac{\tau^{\prime}(t) z^{p-2}(\lambda \tau(t)) z^{\prime}(\lambda \tau(t)) r(t)\left(z^{\prime \prime \prime}(t)\right)^{p-1}}{z^{2(p-1)}(\lambda \tau(t))} .
\end{aligned}
$$

By virtue of Lemma 2.1, we have, for some constant $M>0$ and for all sufficiently large $t$,

$$
z^{\prime}(\lambda \tau(t)) \geq M \tau^{2}(t) z^{\prime \prime \prime}(\tau(t)) \geq M \tau^{2}(t) z^{\prime \prime \prime}(t) .
$$

Combining (2.7) and (2.8), we get

$$
\begin{aligned}
\omega^{\prime}(t) \leq & \rho^{\prime}(t) \frac{r(t)\left(z^{\prime \prime \prime}(t)\right)^{p-1}}{z^{p-1}(\lambda \tau(t))}+\rho(t) \frac{\left(r(t)\left(z^{\prime \prime \prime}(t)\right)^{p-1}\right)^{\prime}}{z^{p-1}(\lambda \tau(t))} \\
& -(p-1) \lambda M \tau^{2}(t) \tau^{\prime}(t) \frac{\rho(t) r(t)\left(z^{\prime \prime \prime}(t)\right)^{p}}{z^{p}(\lambda \tau(t))} .
\end{aligned}
$$

Recalling that $z^{\prime}>0$ and $\sigma(t) \leq t$, we have

$$
x(t)=z(t)-a(t) x(\sigma(t)) \geq z(t)-a(t) z(\sigma(t)) \geq(1-a(t)) z(t) .
$$

Then it follows from (1.1), (2.6), (2.9), and (2.10) that there exists a $t_{3} \geq t_{\lambda}$ such that, for all $t \geq t_{3}$,

$$
\begin{aligned}
\omega^{\prime}(t) \leq & -\rho(t) \sum_{i=1}^{l} q_{i}(t)\left(1-a\left(\tau_{i}(t)\right)\right)^{p-1}+\frac{\rho^{\prime}(t)}{\rho(t)} \omega(t) \\
& -\frac{(p-1) \lambda M \tau^{2}(t) \tau^{\prime}(t)}{(r(t) \rho(t))^{1 /(p-1)}} \omega^{p /(p-1)}(t) .
\end{aligned}
$$


Multiplying the latter inequality by $H(t, s)$ and integrating the resulting inequality from $t_{3}$ to $t$, we obtain

$$
\begin{gathered}
\int_{t_{3}}^{t} H(t, s) \rho(s) \sum_{i=1}^{l} q_{i}(s)\left(1-a\left(\tau_{i}(s)\right)\right)^{p-1} \mathrm{~d} s \\
\leq H\left(t, t_{3}\right) \omega\left(t_{3}\right)+\int_{t_{3}}^{t}\left[\frac{\partial H(t, s)}{\partial s}+\frac{\rho^{\prime}(s)}{\rho(s)} H(t, s)\right] \omega(s) \mathrm{d} s \\
\quad-\int_{t_{3}}^{t} \frac{(p-1) \lambda M \tau^{2}(s) \tau^{\prime}(s)}{(r(s) \rho(s))^{1 /(p-1)}} H(t, s) \omega^{p /(p-1)}(s) \mathrm{d} s \\
\leq H\left(t, t_{3}\right) \omega\left(t_{3}\right)+\int_{t_{3}}^{t} \frac{\varrho_{+}(t, s)}{\rho(s)}(H(t, s))^{(p-1) / p} \omega(s) \mathrm{d} s \\
-\int_{t_{3}}^{t} \frac{(p-1) \lambda M \tau^{2}(s) \tau^{\prime}(s)}{(r(s) \rho(s))^{1 /(p-1)}} H(t, s) \omega^{p /(p-1)}(s) \mathrm{d} s .
\end{gathered}
$$

Now set

$$
A^{p /(p-1)}:=\frac{(p-1) \lambda M \tau^{2}(s) \tau^{\prime}(s)}{(r(s) \rho(s))^{1 /(p-1)}} H(t, s) \omega^{p /(p-1)}(s)
$$

and

$$
B^{1 /(p-1)}:=\frac{(p-1)^{1 / p} \varrho_{+}(t, s)(r(s) \rho(s))^{1 / p}}{p \rho(s)\left(\lambda M \tau^{2}(s) \tau^{\prime}(s)\right)^{(p-1) / p}} .
$$

Letting $\theta:=p /(p-1)$ and using the inequality (see [22])

$$
\theta A B^{\theta-1}-A^{\theta} \leq(\theta-1) B^{\theta}, \quad \theta>1, A \geq 0, B \geq 0,
$$

we have

$$
\begin{aligned}
& \frac{\varrho_{+}(t, s)}{\rho(s)}(H(t, s))^{(p-1) / p} \omega(s)-\frac{(p-1) \lambda M \tau^{2}(s) \tau^{\prime}(s)}{(r(s) \rho(s))^{1 /(p-1)}} H(t, s) \omega^{p /(p-1)}(s) \\
& \quad \leq \frac{1}{p^{p}} \frac{r(s)\left(\varrho_{+}(t, s)\right)^{p}}{\left(\lambda M \tau^{\prime}(s) \tau^{2}(s) \rho(s)\right)^{p-1}} .
\end{aligned}
$$

Hence, we conclude by (2.11) that, for all sufficiently large $t$,

$$
\begin{gathered}
\frac{1}{H\left(t, t_{3}\right)} \int_{t_{3}}^{t}\left[H(t, s) \rho(s) \sum_{i=1}^{l} q_{i}(s)\left(1-a\left(\tau_{i}(s)\right)\right)^{p-1}\right. \\
\left.-\frac{1}{p^{p}} \frac{r(s)\left(\varrho_{+}(t, s)\right)^{p}}{\left(\lambda M \tau^{\prime}(s) \tau^{2}(s) \rho(s)\right)^{p-1}}\right] \mathrm{d} s \leq \omega\left(t_{3}\right),
\end{gathered}
$$

which contradicts (2.3).

Case 3. Assume that (3) is satisfied. We also have (2.10). By virtue of Lemma 2.2, we conclude that, for every constant $k \in(0,1)$, there exists a $t_{k} \geq t_{1}$ such that, for all $t \geq t_{k}$,

$$
z(t) \geq \frac{k}{2} t^{2} z^{\prime \prime}(t)
$$


Now define

$$
\phi(t):=-\delta(t) \frac{r(t)\left(-z^{\prime \prime \prime}(t)\right)^{p-1}}{\left(z^{\prime \prime}(t)\right)^{p-1}}, \quad t \geq t_{1} .
$$

Then $\phi(t)<0$ for all $t \geq t_{1}$. It follows from (1.1), (2.10), (2.13), and (2.14) that there exists a $t_{4} \geq t_{k}$ such that, for all $t \geq t_{4}$,

$$
\begin{aligned}
\phi^{\prime}(t)= & -\delta(t) \sum_{i=1}^{l} q_{i}(t)\left(1-a\left(\tau_{i}(t)\right)\right)^{p-1} \frac{z^{p-1}(\tau(t))}{\left(z^{\prime \prime}(\tau(t))\right)^{p-1}} \frac{\left(z^{\prime \prime}(\tau(t))\right)^{p-1}}{\left(z^{\prime \prime}(t)\right)^{p-1}} \\
& +\frac{\delta^{\prime}(t)}{\delta(t)} \phi(t)-(p-1) \frac{(-\phi(t))^{p /(p-1)}}{(r(t) \delta(t))^{1 /(p-1)}} \\
\leq & -\delta(t)\left(\frac{k \tau^{2}(t)}{2}\right)^{p-1} \sum_{i=1}^{l} q_{i}(t)\left(1-a\left(\tau_{i}(t)\right)\right)^{p-1} \\
& +\frac{\delta^{\prime}(t)}{\delta(t)} \phi(t)-(p-1) \frac{(-\phi(t))^{p /(p-1)}}{(r(t) \delta(t))^{1 /(p-1)}} .
\end{aligned}
$$

Multiplying (2.15) by $K(t, s)$ and integrating the resulting inequality from $t_{4}$ to $t$, we obtain

$$
\begin{aligned}
\int_{t_{4}}^{t} K(t, s) \delta(s)\left(\frac{k \tau^{2}(s)}{2}\right)^{p-1} \sum_{i=1}^{l} q_{i}(s)\left(1-a\left(\tau_{i}(s)\right)\right)^{p-1} \mathrm{~d} s \\
\leq K\left(t, t_{4}\right) \phi\left(t_{4}\right)+\int_{t_{4}}^{t}\left[\frac{\partial K(t, s)}{\partial s}+\frac{\delta^{\prime}(s)}{\delta(s)} K(t, s)\right] \phi(s) \mathrm{d} s \\
\quad-(p-1) \int_{t_{4}}^{t} K(t, s) \frac{(-\phi(s))^{p /(p-1)}}{(r(s) \delta(s))^{1 /(p-1)}} \mathrm{d} s \\
\leq K\left(t, t_{4}\right) \phi\left(t_{4}\right)-\int_{t_{4}}^{t} \frac{\xi_{+}(t, s)}{\delta(s)}(K(t, s))^{(p-1) / p} \phi(s) \mathrm{d} s \\
-(p-1) \int_{t_{4}}^{t} K(t, s) \frac{(-\phi(s))^{p /(p-1)}}{(r(s) \delta(s))^{1 /(p-1)}} \mathrm{d} s .
\end{aligned}
$$

Set

$$
A^{p /(p-1)}:=(p-1) K(t, s) \frac{(-\phi(s))^{p /(p-1)}}{(r(s) \delta(s))^{1 /(p-1)}}
$$

and

$$
B^{1 /(p-1)}:=\frac{(p-1)^{1 / p}(r(s) \delta(s))^{1 / p} \xi_{+}(t, s)}{p \delta(s)} .
$$

Letting $\theta:=p /(p-1)$ and using inequality (2.12), we have by (2.16) that, for all sufficiently large $t$,

$$
\begin{aligned}
& \int_{t_{4}}^{t}\left[K(t, s) \delta(s)\left(\frac{k \tau^{2}(s)}{2}\right)^{p-1} \sum_{i=1}^{l} q_{i}(s)\left(1-a\left(\tau_{i}(s)\right)\right)^{p-1}-\frac{r(s)\left(\xi_{+}(t, s)\right)^{p}}{p^{p} \delta^{p-1}(s)}\right] \mathrm{d} s \\
& \quad \leq K\left(t, t_{4}\right) \phi\left(t_{4}\right)<0,
\end{aligned}
$$

which contradicts (2.4). This completes the proof. 
Remark 2.4 Choosing different combinations of functions $H, \rho, K$, and $\delta$, one can derive from Theorem 2.3 a variety of efficient tests for oscillation of equation (1.1) and its particular cases.

\section{Example and discussion}

The following example illustrates applications of Theorem 2.3.

Example 3.1 For $t \geq 1$ and $0 \leq a_{0}<1$, consider the fourth-order neutral differential equation

$$
\left(t^{2}\left(x(t)+a_{0} x(t-2 \pi)\right)^{\prime \prime \prime}\right)^{\prime}+\left(1+a_{0}\right) t^{2} x(t-3 \pi)+2\left(1+a_{0}\right) t x\left(t+\frac{\pi}{2}\right)=0 .
$$

Let $p=2, \tau(t)=t-3 \pi, \rho(t)=\delta(t)=1$, and $H(t, s)=K(t, s)=(t-s)^{2}$. It is not difficult to verify that all assumptions of Theorem 2.3 are satisfied, and hence equation (3.1) is oscillatory. As a matter of fact, one such solution is $x(t)=\sin t$.

Remark 3.2 Oscillation theorem established in this paper for equation (1.1) complements, on one hand, results reported by Baculíková and Džurina [6], Karpuz [7], and Li et al. [9] because we use assumption (1.3) rather than (1.2) and, on the other hand, those by Li et al. [10] and Zhang et al. [16, 17, 19-21] since our theorem can be applied to the case where $a(t) \neq 0$.

Remark 3.3 We point out that, contrary to [11, Theorem 2.2], Theorem 2.3 does not need restrictive conditions (1.4) and can ensure that all solutions of equation (1.1) oscillate, which, in a certain sense, is a significant improvement compared to [11, Theorem 2.2] for fourth-order neutral differential equations.

Remark 3.4 It would be of interest to study equation (1.1) in the case where

$$
\int_{t_{0}}^{\infty} \int_{u}^{\infty} R(s) \mathrm{d} s \mathrm{~d} u<\infty
$$

for future research.

\section{Competing interests}

The authors declare that they have no competing interests.

Authors' contributions

All authors contributed equally to this work. They all read and approved the final version of the manuscript.

\section{Author details}

'Qingdao Technological University, Feixian, Shandong 273400, P.R. China. ${ }^{2}$ Department of Mathematics, Faculty of Electrical Engineering and Informatics, Technical University of Košice, Letná 9, Košice, 042 00, Slovakia. ${ }^{3}$ School of Control Science and Engineering, Shandong University, Jinan, Shandong 250061, P.R. China.

\section{Acknowledgements}

The authors express their sincere gratitude to the anonymous referees for the careful reading of the original manuscript and useful comments that helped to improve the presentation of the results and accentuate important details. This research is supported by the National Key Basic Research Program of P.R. China (2013CB035604) and NNSF of P.R. China (Grant Nos. 61034007, 51277116, 51107069). 


\section{References}

1. Aronsson, G, Janfalk, U: On Hele-Shaw flow of power-law fluids. Eur. J. Appl. Math. 3, 343-366 (1992)

2. Bartušek, M, Cecchi, M, Došlá, Z, Marini, M: Fourth-order differential equation with deviating argument. Abstr. Appl. Anal. 2012, 185242 (2012)

3. Agarwal, RP, Bohner, M, Li, W-T: Nonoscillation and Oscillation: Theory for Functional Differential Equations. Dekker, New York (2004)

4. Agarwal, RP, Grace, SR, O'Regan, D: Oscillation Theory for Difference and Functional Differential Equations. Kluwer Academic, Dordrecht (2000)

5. Agarwal, RP, Grace, SR, O'Regan, D: Oscillation criteria for certain $n$th order differential equations with deviating arguments. J. Math. Anal. Appl. 262, 601-622 (2001)

6. Baculíková, B, Džurina, J: Oscillation theorems for higher order neutral differential equations. Appl. Math. Comput. 219, 3769-3778 (2012)

7. Karpuz, B: Sufficient conditions for the oscillation and asymptotic behaviour of higher-order dynamic equations of neutral type. Appl. Math. Comput. 221, 453-462 (2013)

8. Kiguradze, IT, Chanturia, TA: Asymptotic Properties of Solutions of Nonautonomous Ordinary Differential Equations. Kluwer Academic, Dordrecht (1993). Translated from the 1985 Russian original

9. Li, T, Han, Z, Zhao, P, Sun, S: Oscillation of even-order neutral delay differential equations. Adv. Differ. Equ. 2010, $184180(2010)$

10. Li, T, Thandapani, E, Tang, S: Oscillation theorems for fourth-order delay dynamic equations on time scales. Bull. Math Anal. Appl. 3, 190-199 (2011)

11. Li, T, Zhang, C, Thandapani, E: Asymptotic behavior of fourth-order neutral dynamic equations with noncanonical operators. Taiwan. J. Math. (2014). doi:10.11650/tjm.18.2014.2678

12. Parhi, N, Tripathy, AK: On oscillatory fourth order nonlinear neutral differential equations. I. Math. Slovaca 54, 389-410 (2004)

13. Parhi, N, Tripathy, AK: On oscillatory fourth order nonlinear neutral differential equations. II. Math. Slovaca 55, 183-202 (2005)

14. Philos, ChG: A new criterion for the oscillatory and asymptotic behavior of delay differential equations. Bull. Acad. Pol. Sci., Sér. Sci. Math. 39, 61-64 (1981)

15. Thandapani, E, Savitri, R: Oscillation and nonoscillation of fourth-order nonlinear neutral differential equations. Indian J. Pure Appl. Math. 32, 1631-1642 (2001)

16. Zhang, C, Agarwal, RP, Bohner, M, Li, T: New results for oscillatory behavior of even-order half-linear delay differential equations. Appl. Math. Lett. 26, 179-183 (2013)

17. Zhang, C, Agarwal, RP, Bohner, M, Li, T: Properties of higher-order half-linear functional differential equations with noncanonical operators. Adv. Differ. Equ. 2013, 54 (2013)

18. Zhang, C, Agarwal, RP, Li, T: Oscillation and asymptotic behavior of higher-order delay differential equations with p-Laplacian like operators. J. Math. Anal. Appl. 409, 1093-1106 (2014)

19. Zhang, C, Li, T, Agarwal, RP, Bohner, M: Oscillation results for fourth-order nonlinear dynamic equations. Appl. Math Lett. 25, 2058-2065 (2012)

20. Zhang, C, Li, T, Saker, SH: Oscillation of fourth-order delay differential equations. Nonlinear Oscil. 16, $322-335$ (2013)

21. Zhang, C, Li, T, Sun, B, Thandapani, E: On the oscillation of higher-order half-linear delay differential equations. Appl. Math. Lett. 24, 1618-1621 (2011)

22. Hardy, GH, Littlewood, JE, Polya, G: Inequalities. Cambridge University Press, Cambridge (1988)

$10.1186 / 1687-2770-2014-56$

Cite this article as: Li et al.: Oscillation of fourth-order neutral differential equations with $p$-Laplacian like operators.

Boundary Value Problems 2014, 2014:56

\section{Submit your manuscript to a SpringerOpen ${ }^{\circ}$ journal and benefit from:}

- Convenient online submission

- Rigorous peer review

- Immediate publication on acceptance

- Open access: articles freely available online

- High visibility within the field

- Retaining the copyright to your article 